\title{
Establishing Glaucoma Clinic: Essential or Nonessential For General Hospital
}

\author{
Dr. Shyam Kumar Gupta ${ }^{1}$, Dr. Praveen Malik ${ }^{2}$, Dr. Bhaskar Agarwal ${ }^{3 *}$
}

${ }^{1}$ (MBBS, MS), Postgraduate Institute of Medical Education and Research (Dr. RML Hospital), New Delhi, India

${ }^{2}$ Professor \& Head, Department of Opthalmology, Postgraduate Institute of Medical Education and Research (Dr. RML Hospital), New Delhi, India ${ }^{3}$ Associate Professor, Department of Prosthodontics, King George's Medical University, Shah Mina Rd, Chowk, Lucknow, Uttar Pradesh 226003, India

*Corresponding author: Dr. Bhaskar Agarwal

Abstract

Introduction: Since India is a developing country, our health infrastructure is not well standardised at every hospital. Some hospitals are providing all documentation and special care to glaucoma patients by establishing Glaucoma Clinics (GC), but unfortunately their numbers are very less in our country. So we want to study the beneficial impact of GC in glaucoma patient treatment. Material and methods: All those patients' records who are diagnosed as glaucoma and being treated for the same in glaucoma clinic or in routine OPD for a minimum period of 2 years are analyzed in the study. Results: 1298 patients from glaucoma clinic and 710 patients from routine OPD fulfilled the inclusion criteria of the study. Out of these 710 OPD patients 143 patients $(20.1 \%)$ did not have glaucoma based on evaluation in our glaucoma clinic. $1268(97.7 \%)$ glaucoma clinic patient out of 1298 have achieved the target IOP, whereas 489 (86.2\%) OPD patients out of total 567 which is statically significant $\mathrm{P}$ valve $<0.0001$. Glaucoma progression was $1 \%$ in Glaucoma Clinic group in compression to $13 \%$ in OPD group which is statically insignificant p valve 0.028. Discussion: A good adherence and persistency to the treatment is necessary but cost, misbelieve, non communication between doctor and patients are major obstruction. By establishing G C better information, communication and education can be given that's the reason of higher follow up compliance and adherence than OPD patients and previous studies. Conclusion: Glaucoma is a sight threatening disease and the number of such patient is increasing day by day. So to negotiate the upcoming health threat we should surely focus on establishing the specialized glaucoma clinics.

Keywords: Glaucoma, Optic Nerve, Glaucoma clinic.

Copyright $\left({ }_{0} 2021\right.$ The Author(s): This is an open-access article distributed under the terms of the Creative Commons Attribution 4.0 International License (CC BY-NC 4.0) which permits unrestricted use, distribution, and reproduction in any medium for non-commercial use provided the original author and source are credited.

\section{INTRODUCTION}

Glaucoma is a sight threatening disease. It usually needs long term follow-up and management. For good glaucoma management documentation of the base line and follow-up is mandatory. Goal of glaucoma Therapy to preserve the vision related quality of life of the patient so that the patient does not develop any functional disability in his/her life span. The specific goals of therapy are: To achieve target IOP and minimise fluctuations with minimum medicine, To educate and involve the patient and his family in the management of the disease process, To monitor the progression in reference to structure and function of the optic nerve damage and dynamically adjust the target IOP, To select the medicines which can provide the desired effect with least side effects and minimum effect on quality of life, To achieve treatment at an affordable and sustainable cost and To treat systemic factors possibly affecting the optic nerve [1-3].
Since India is a developing country, our health infrastructure is not well standardised at every hospital. Some hospitals are providing all documentation and special care to glaucoma patients by establishing Glaucoma Clinics (GC), but unfortunately their numbers are very less in our country. So we want to study the beneficial impact of GC in glaucoma patient treatment. Compliance to Follow Ups was $97.8 \%$ in GC group in compression to $76.2 \%$ in OPD patients group which has $\mathrm{p}$ valve $<0.001$, statically significant. Adherence to treatment was $91.7 \%$ in GC group as compassion to $76.7 \%$ in OPD group which is statically significant $p$ value $<0.001$. Information, Communication and Education for glaucoma was $100 \%$ in GC group and $47.9 \%$ in OPD group which is statically significant $\mathrm{p}$ value $<0.001$. Availability of base line \& follow ups record $1298(100 \%)$ and $240(42.32 \%)$ in OPD group which is statically significant $\mathrm{p}$ value $<0.001$. Target IOP mentioned on the $779(60 \%)$ in GC and zero in OPD group which is statically significant $\mathrm{p}$ value $<0.001$. 
Shyam Kumar Gupta et al., Sch Acad J Biosci, Feb, 2021; 9(2): 22-24

Diurnal variation mentioned on the records 1233 (95\%) in GC group and zero in OPD group which is statically significant $\mathrm{p}$ value $<0.001$. $^{1,3,4,5}$

Study Design: A retrospective medical audit study.

Aims: To compare the quality of management of glaucoma patients in glaucoma clinic Vs general ophthalmic OPD.

\section{MATERIAL AND METHODS}

We conducted the study at PGIMER (Dr. R M L Hospital) New Delhi. It's a retrospective study. All those patients' records who were diagnosed as glaucoma and being treated for the same in glaucoma clinic or in routine OPD for a minimum period of 2 years are analyzed in the study. The file records were noted under Primary Outcome Variables as:

1) Target IOP achieved or not at the time of enrollment.

2) Any Disease progression- based on automated perimetery/Optic N. head records changes.
Besides these we also noted the following secondary Outcome variables as per European Glaucoma society guidelines [6]. Which are as follows:

1. Compliance to follow up

2. Adherence to treatment

3. Availability of baseline and follow up records

4. Information Education and Communication for glaucoma

5. Diurnal variation and target IOP

Those patients, who did not come even on single visit, in the least 3 years, were excluded from the study. All the outcome parameters were analyzed by using Chi square test.

\section{RESULTS}

1298 patients from glaucoma clinic and 710 patients from routine OPD fulfilled the inclusion criteria of the study. Out of these 710 OPD patients 143 patients $(20.1 \%)$ did not have glaucoma based on evaluation in our glaucoma clinic. So, only 567 OPD patients were enrolled in our study.

Table-1: Primary variables

\begin{tabular}{|l|l|l|l|}
\hline Outcome measures & Glaucoma Clinic & OPD & P value \\
\hline Achieved Target IOP & $1268(97.7 \%)$ & $489(86.2 \%)$ & $<.0001$ \\
\hline Glaucoma progression & $13(1.0 \%)$ & $13(2.3 \%)$ & .028 \\
\hline Total & $\mathbf{1 2 9 8}$ & $\mathbf{5 6 7}$ & \\
\hline
\end{tabular}

$1268(97.7 \%)$ glaucoma clinic patient out of 1298 have achieved the target IOP, whereas 489 (86.2\%) OPD patients out of total 567 which is statically significant $\mathrm{P}$ valve $<0.0001$. Glaucoma progression was
$1 \%$ in Glaucoma Clinic group in compression to $13 \%$ in OPD group which is statically insignificant $\mathrm{p}$ valve 0.028 .

Table-2: Secondary variables

\begin{tabular}{|l|l|l|l|}
\hline Outcome measures & Glaucoma Clinic & OPD & P value \\
\hline Compliance to Follow Ups & $1270(97.8 \%)$ & $541(76.2 \%)$ & $<.0001$ \\
\hline Adherence to Treatment & $1190(91.7 \%)$ & $545(76.7 \%)$ & $<.0001$ \\
\hline ICE for glaucoma & $1298(100 \%)$ & $340(47.9 \%)$ & $<.0001$ \\
\hline Availability of base line \& follow ups record & $1298(100 \%)$ & $240(42.32 \%)$ & $<.0001$ \\
\hline Target IOP mentioned on the records & $779(60 \%)$ & $0(0 \%)$ & 0.00 \\
\hline Diurnal variation mentioned on the records & $1233(95 \%)$ & $0(0 \%)$ & 0.00 \\
\hline
\end{tabular}

Compliance to Follow Ups was $97.8 \%$ in GC group in compression to $76.2 \%$ in OPD patients group which has $\mathrm{p}$ valve $<0.001$, statically significant. Adherence to treatment was $91.7 \%$ in GC group as compassion to $76.7 \%$ in OPD group which is statically significant $p$ value $<0.001$. Information, Communication and Education for glaucoma was $100 \%$ in GC group and $47.9 \%$ in OPD group which is statically significant $\mathrm{p}$ value $<0.001$. Availability of base line \& follow ups record $1298(100 \%)$ and $240(42.32 \%)$ in OPD group which is statically significant $\mathrm{p}$ value $<0.001$. Target IOP mentioned on the $779(60 \%)$ in GC and zero in OPD group which is statically significant $\mathrm{p}$ value $<0.001$. Diurnal variation mentioned on the records 1233 (95\%) in GC group and zero in OPD group which is statically significant $\mathrm{p}$ value $<0.001$

\section{DISCUSSION}

A good adherence and persistency to the treatment is necessary but cost, misbelieve, non communication between doctor and patients are major obstruction. By establishing G C better information, communication and education can be given that's the reason of higher follow up compliance and adherence than OPD patients and previous studies [1-5]. 
Other benefits of $\mathrm{G} \mathrm{C}$

1. Most crucial face to face discussion among patients can't be given by any other means

2. These records can help us to modify our management strategy as most of these are based on western population studies

3. Useful source of material for research

\section{CONCLUSION}

Establishment of Glaucoma Clinics should be seriously considered by the health care providers and policy makers especially in government sector where generally no records of OPD patients are kept.

\section{REFERENCE}

1. Morley AM, Murdoch I. The future of glaucoma clinics. Br J Ophthalmol. 2006; 90(5):640-5.
2. Bowling B, Chen SD, Salmon JF. Outcomes of referrals by community optometrists to a hospital glaucoma service. $\mathrm{Br}$ J Ophthalmol. 2005; 89(9):1102-4.

3. Bettin P, Di Matteo F. Glaucoma: present challenges and future trends. Ophthalmic Res. 2013; 50(4):197-208.

4. Eni EN, Edet BE, Ibanga AA, Duke RE. Cross-Sectional Study on the Coping Strategies Among Glaucoma Patients Attending a Secondary Eye Clinic in Calabar, Nigeria. Clin Ophthalmol. 2020; 14:1307-1313.

5. Guo CY, Qi XH, Qi JM. Systematic review and Meta-analysis of treating open angle glaucoma with gonioscopy-assisted transluminal trabeculotomy. Int J Ophthalmol. 2020; 13(2):317-324.

6. European Glaucoma society guidelines $5^{\text {th }}$ ed. https://www.eugs.org/eng/guidelines.asp 